\title{
RESIDUOS ORGANOCLORADOS EN TEJIDO ADIPOSO MAMARIO Y SU RELACIÓN CON EL CÁNCER DE MAMA
}

\author{
ORGANOCHLORATE RESIDUES IN MAMMARY ADIPOSE TISSUE \\ AND THEIR RELATION WITH TO BREAST CANCER
}

ljoni Costabeber ${ }^{1}$; Rosario Angulo ${ }^{2} \&$ Manuela Jodral $^{3}$

\begin{abstract}
'Dra em Ciência e Tecnologia dos Alimentos. Departamento de Tecnologia e Ciência dos Alimentos, Centro de Ciências Rurais, Universidade Federal de Santa Maria. '2Dra en Medicina y en Ciencia y Tecnología de los Alimentos. Departamento de Medicina Preventiva y Salud Pública, Facultad de Medicina, Universidad de Córdoba, España. ${ }^{3}$ Dra en Veterinaria. Departamento de Bromatología y Tecnología de los Alimentos, Facultad de Veterinaria, Universidad de Córdoba, España.

Campus Universitario de Camobi, Santa Maria, RS. Brasil. FAX: (055) 220-8353 - CEP 97105-900. E-mail: ihcbeber@alunop.ufsm.br.
\end{abstract}

COSTABEBER I; ANGULO R \& JODRAL M. Residuos organoclorados en tejido adiposo mamario y su relación con el cáncer de mama. Medicina, Ribeirão Preto, 33: 506-514, oct./dic. 2000.

RESUMEN: Teniendo en cuenta la repercusión sanitaria que residuos organoclorados puedan tener, se determinaron niveles de pesticidas organoclorados y bifenilos policlorados (PCBs) en muestras de tejido adiposo mamario de donantes que padecían cáncer de mama (grupo con patología maligna) y de donantes que presentaban alteraciones benignas en las mamas (grupo control). Además, se estudiaron datos epidemiológicos de estas mujeres y, posteriormente, se estudió la relación residuos organoclarados/datos epidemiológicos. Los análisis indicaron que las concentraciones de Aldrín, PCB $n^{\circ} 28$ y PCB $n^{\circ} 52$ fueron estadísdicamente diferentes entre los dos grupos de donantes. Los niveles de estos compuestos fueron mayores en el grupo con patología maligna que en el grupo control. Ello sugiere la posibilidad de una relación positiva entre la presencia de estos residuos en las muestras analizadas y la malignidad de los tumores en las mamas de las donantes.

PALAVRAS CLAVE: Residuos Organoclorados. Cáncer de Mama.

\section{1- INTRODUCCIÓN}

Actualmente, hay un creciente interés público y científico respecto a los contaminantes ambientales y sus implicaciones sobre la incidencia de enfermedades neoplásicas en humanos. Investigaciones como las realizadas por Unger et al. (1984) $)^{(1)}$, MussaloRauhamaa et al. $(1990)^{(2)}$, Falck et al. $(1992)^{(3)}$, Krieger et al. (1994) ${ }^{(4)}$ y Costabeber $(1999)^{(5)}$ muestran que residuos organoclorados pueden estar asociados con la incidencia de cáncer de mama en mujeres, si bien algunos autores señalan que la relación entre estos compuestos y la salud es todavía poco clara ${ }^{(6,7,8)}$. En este contexto, el objetivo general de este trabajo fue el de realizar la identificación cualitativa y cuantitativa de residuos organoclorados en tejido graso mamario, así como conocer la posible repercusión sanitaria que dichos compuestos puedan tener sobre la salud de la población femenina. Para ello, se estudiaron datos epidemiológicos y muestras de tejido adiposo mamario de mujeres, residentes en la provincia de Córdoba (España), que fueron intervenidas quirúrgicamente en la Unidad de Patología Mamaria de la Ciudad Sanitaria Reina Sofía. 


\section{2- MATERIAL Y MÉTODO}

Por medio de cuestionarios (encuestas) se recopilaron datos personales de las donantes, tales como: edad, número de hijos, meses de lactancia, edad primer parto, número de abortos, edad menarquia y menopausia, peso, talla, zona de nacimiento y domicilio, antecedentes familiares y personales, estado civil, ocupación laboral anterior y actual, hábitos fumadores y alcohólicos, empleo de productos en agricultura y ganadería, y datos referentes a hábitos alimentarios (alimento más consumido y consumo de pescado, carne, leche, frutas, verduras y legumbres). También se recopilaron datos referentes a la procedencia de la muestra, el volumen de la mama y la localización de la lesión.

Los informes resultantes de los estudios anatomopatológicos de las donantes, realizados por el equipo médico del hospital, permitieron la clasificación de los datos de los cuestionarios y de las 134 muestras de tejido mamario en dos grupos: el grupo control y el grupo con patología maligna. Las donantes cuyas lesiones anatomopatológicas fueron informadas como fibroadenoma, mastopatía fibroquística y "otras alteraciones" en las mamas fueron clasificadas en el "grupo control", y las que presentaron informes anatomopatológicos de lesiones carcinomatosas en las mamas fueron clasificadas en el "grupo con patologías malignas". La clase "otras alteraciones" se refiere a las donantes que presentaron alteraciones tales como: adenosis, fibrosis, ectasia ductal, mastitis, granuloma lipofágico, necrosis grasa, papiloma intraductal y tumor filodes benigno.

La Gráfica I presenta la distribución de frecuencia de las clases de muestras objeto de investigación, teniéndose en cuenta el informe anatomo-patológico. El grupo control lo formaron 65 donantes, lo que corresponde a un $48,5 \%$ de las muestras. El grupo de donantes con patologías malignas estuvo integrado por 69 mujeres, lo que corresponde a un $51,5 \%$ de las muestras analizadas.

Se ha puesto a punto $\mathrm{y}$ validado una técnica multirresiduo para la investigación de los residuos de cuatro pesticidas organoclorados (HCB (hexaclorobenceno), $\gamma$-HCH (lindano), Aldrín y p,p'DDE) y de once congéneres de bifenilos policlorados $\left(\right.$ n $^{\text {os. }} 28,52,101,118,138,153,170,180,183,187$ y 188 ) en tejido adiposo. Las muestras fueron sometidas a un proceso de extracción y purificación con $n$-hexano y florisil, según la metodología de Garrido et al. $(1992)^{(9)}$. El método empleado permitió la obtención de extractos limpios que fueron analizados por cromatógrafo de gases con detector de captura de electrones. Mediante el proceso de validación, se ha demostrado la correcta linealidad, precisión, exactitud y sensibilidad del método analítico empleado.

Para el análisis estadístico de los resultados obtenidos se ha empleado los programas informáticos Statistica versión 6.0 y Statistical Analysis System (SAS) versión 6.1. Para los datos referentes a las concentraciones de los residuos organoclorados y de las encuestas, se realizaron las siguientes pruebas: a) estadísticos básicos (media, desviación típica, mínimos y máximos), b) coeficientes de correlación lineal simple, c) distribución de frecuencias del total de muestras analizadas y muestras según los grupos de donantes, con su $\chi^{2}$ de asociación y, d) prueba $\mathbf{t}$ de Student.

\section{3- RESULTADOS Y DISCUSIÓN}

Los resultados obtenidos son presentados en los puntos siguientes: a) residuos organoclorados, b) datos de las encuestas y, c) relación datos encuestas/ residuos organoclorados.

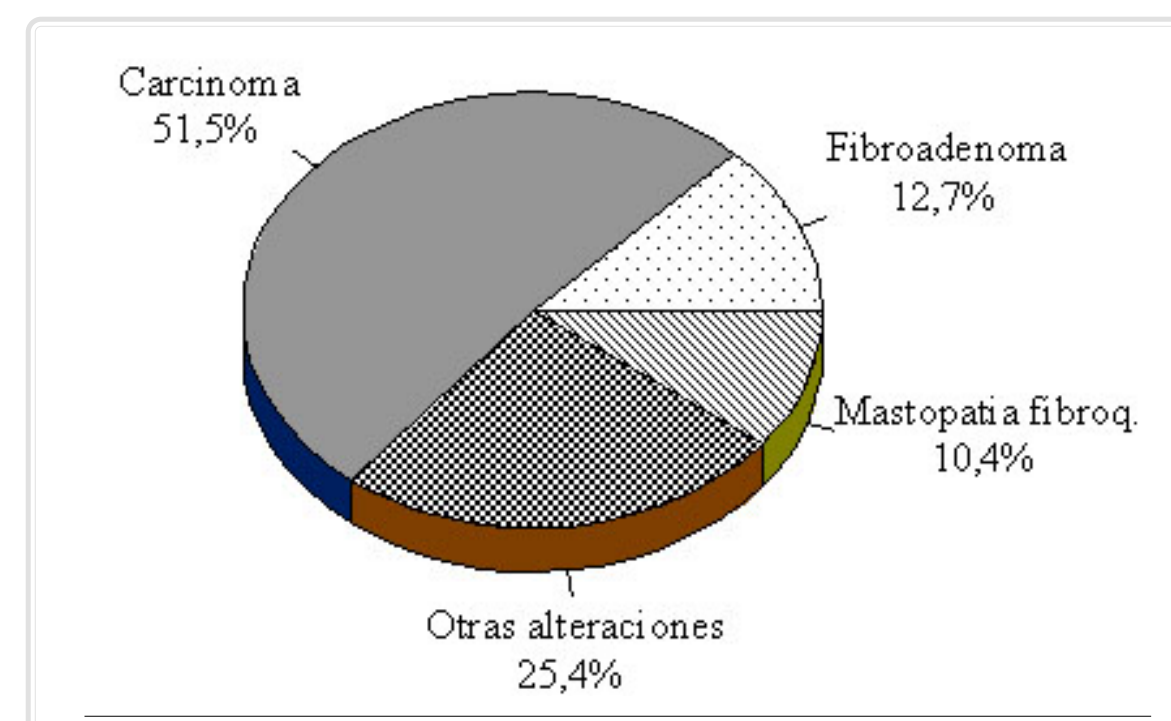

Gráfica I - Informe Anatomopatológico de las donantes 


\section{a) Residuos organoclorados}

Los resultados alcanzados en la investigación sobre los residuos organoclorados en las muestras analizadas se encuentran recogidos en la Tabla I.

De entre los pesticidas organoclorados, el p,p'DDE fue detectado en todas las muestras analizadas y en niveles superiores a los demás compuestos investigados. Kanja et al. (1992)(10), Ludwicki y Góralczyk (1994) ${ }^{(11)}$, Gallelli et al. (1995) ${ }^{(12)}$, Waliszewski et al. (1995) $)^{(13)}$ y Kamarinos et al. $(1997)^{(14)}$ detectaron también el p,p'-DDE en todas las muestras analizadas, además dicho compuesto presentaba niveles medios más elevados cuando los compararon con los niveles medios detectados de otros compuestos investigados. El HCB, Aldrín y $\gamma-\mathrm{HCH}$ presentaron frecuencias de un $98 \%$, un $64 \%$ y un $16 \%$, respectivamente. Los niveles medios detectados fueron de $1,87 \mu \mathrm{g} / \mathrm{g}$ de tejido mamario para el p,p'-DDE, 0,239 $\mu \mathrm{g} / \mathrm{g}$ para el HCB, $0,009 \mu \mathrm{g} / \mathrm{g}$ para el Aldrín y 0,018 $\mu \mathrm{g} / \mathrm{g}$ para el $\gamma-\mathrm{HCH}$. Se observó que los pesticidas organoclorados, excepto el p,p'-DDE presentaron niveles más bajos en el grupo control que en el grupo con patología maligna.

Respecto a los bifenilos policlorados (PCBs), los congéneres $\mathrm{n}^{\text {os. }} 138,153,170,180$ y 187 tuvieron una frecuencia de un $100 \%$ en las muestras de tejido adiposo. Se identifican los congéneres de PCBs $n^{\text {os. }}$ 118,183 y 188 en un $90 \%$ de las muestras, y los congéneres $\mathrm{n}^{\text {os. }} 28,52$ y 101 en menores porcentajes. Los bifenilos policlorados, en su conjunto, presentaron un nivel medio de $0,6 \mu \mathrm{g} / \mathrm{g}$ de tejido mamario. Este valor fue ligeramente inferior a la concentración media de $0,66 \mu \mathrm{g} / \mathrm{g}$ de tejido subcutáneo encontrada por Skaare et al. (1988) ${ }^{(15)}$; al nivel medio de $0,75 \mu \mathrm{g} / \mathrm{g}$ de tejido adiposo determinado por Duarte-Davidson et al. $(1993)^{(16)}$ y al valor de $0,79 \mu \mathrm{g} / \mathrm{g}$ de tejido adiposo detectado por Duarte-Davidson et al. (1994) ${ }^{(17)}$. Una concentración media más elevada $(1,4 \mu \mathrm{g} / \mathrm{g}$ de tejido adiposo) que la encontrada en este estudio fue determinada por Poli et al. (1995) ${ }^{(18)}$ en las muestras de tejido adiposo de residentes en el Distrito de Trento

\begin{tabular}{|c|c|c|c|c|}
\hline \multirow[b]{2}{*}{ Compuestos } & \multirow{2}{*}{$\begin{array}{l}\text { Frecuencia de } \\
\text { determinación }\end{array}$} & \multirow{2}{*}{$\begin{array}{c}\text { Va lores } \\
\text { Total de muestras } \\
\text { analizadas }\end{array}$} & \multicolumn{2}{|c|}{ M é d i os $\quad(\mu \mathrm{g} / \mathrm{g}$ de tejido adiposo } \\
\hline & & & Grupo control & $\begin{array}{c}\text { Grupo con patología } \\
\text { maligna }\end{array}$ \\
\hline \multicolumn{5}{|l|}{ Pesticidas } \\
\hline HCB & 98,51 & 0,239 & 0,207 & 0,263 \\
\hline$\gamma-\mathrm{HCH}$ & 15,67 & 0,018 & 0,001 & 0,004 \\
\hline Aldrin & 64,18 & 0,009 & 0,002 & 0,008 \\
\hline$p, p^{\prime}-\mathrm{DDE}$ & 100,00 & 1,869 & 2,099 & 1,652 \\
\hline \multicolumn{5}{|l|}{ Bifenilos } \\
\hline PCB no 28 & 61,94 & 0,062 & 0,016 & 0,059 \\
\hline PCB n 52 & 66,42 & 0,017 & 0,007 & 0,014 \\
\hline PCB no 101 & 49,25 & 0,005 & 0,002 & 0,003 \\
\hline PCB no 118 & 93,28 & 0,025 & 0,024 & 0,022 \\
\hline PCB no 188 & 97,01 & 0,011 & 0,011 & 0,011 \\
\hline PCB no 153 & 100,00 & 0,122 & 0,126 & 0,119 \\
\hline PCB no 138 & 100,00 & 0,103 & 0,107 & 0,099 \\
\hline PCB no 187 & 100,00 & 0,047 & 0,050 & 0,045 \\
\hline PCB no 183 & 94,78 & 0,021 & 0,021 & 0,019 \\
\hline PCB no 180 & 100,00 & 0,137 & 0,142 & 0,131 \\
\hline PCB no 170 & 100,00 & 0,046 & 0,048 & 0,045 \\
\hline
\end{tabular}


(Italia). Las concentra-ciones medias más altas detectadas en las muestras de las donantes cordobesas correspon-dieron al congénere $\mathrm{n}^{\circ} 180(0,137 \mu \mathrm{g} / \mathrm{g}$ de tejido mamario), seguido de los congéneres $\mathrm{n}^{\circ} 153$ $(0,122 \mu \mathrm{g} / \mathrm{g})$ y $^{\mathrm{o}} 138(0,103 \mu \mathrm{g} / \mathrm{g})$. Las concentraciones medias más bajas correspon-dieron a los congéneres $\mathrm{n}^{\circ} 101(0,005 \mu \mathrm{g} / \mathrm{g}$ de tejido mamario), $\mathrm{n}^{\circ} 188(0,011 \mu \mathrm{g} / \mathrm{g})$ y n $^{\circ} 52(0,017 \mu \mathrm{g} / \mathrm{g})$.

Las concentraciones medias de los residuos de PCBs detectadas en las muestras fueron ligeramente superiores en el grupo con patología maligna. Cuando se comparan los niveles medios de los congé-neres individuales en los dos grupos, se observa que los congéneres $\mathrm{n}^{\text {os. }} 28,52$ y 101 presentaron concen- traciones medias más bajas en las muestras controles, mientras los congéneres $\mathrm{n}^{\text {os. }} 118,138,153,170,180$, 183 y 187 presentaron valores medios más bajos en las muestras del grupo con patología maligna.

Los niveles de Aldrín, PCB $n^{\circ} 28$ y PCB $n^{\circ} 52$ son estadísticamente diferentes $(\mathrm{p}<0,01)$ en los grupos control y con patología maligna. Las Gráficas II, III y IV muestran la distribución de frecuencia de las muestras según los valores detectados de estos compuestos en las muestras de donantes controles y de donantes con patología maligna. Las concentraciones medias de estos compuestos fueron menores en el grupo control que en el grupo con patología maligna, y ello sugiere la posibilidad de una relación positiva en-

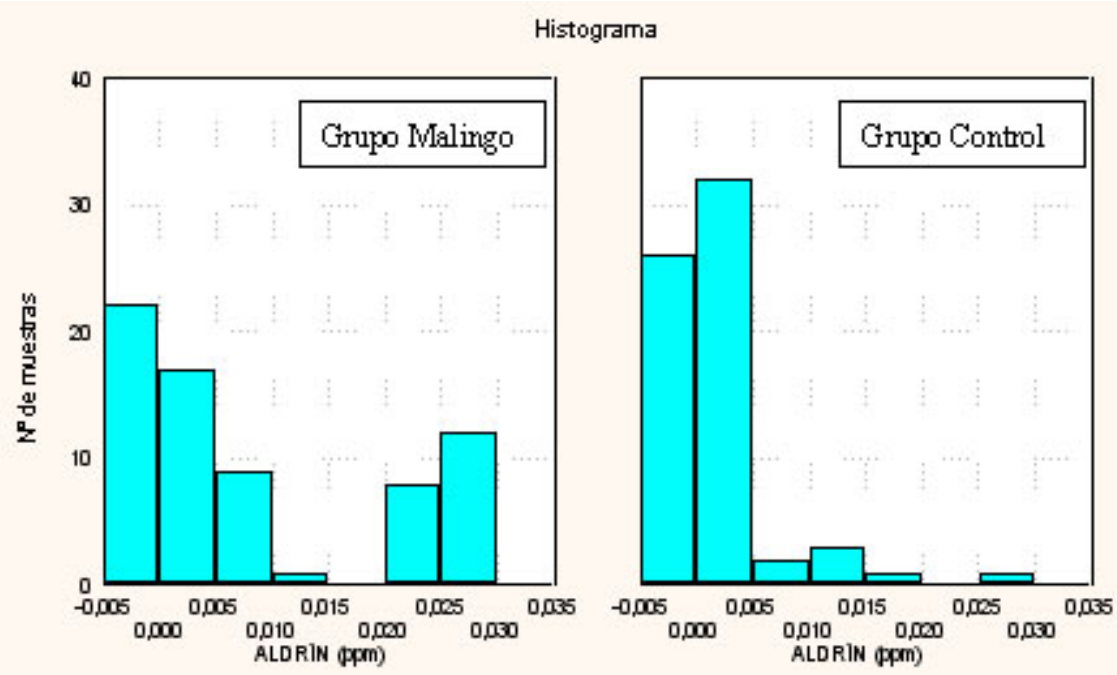

Gráfica II - Distribución de frecuencia de las muestras según los grupos y concentraciones de Aldrín.

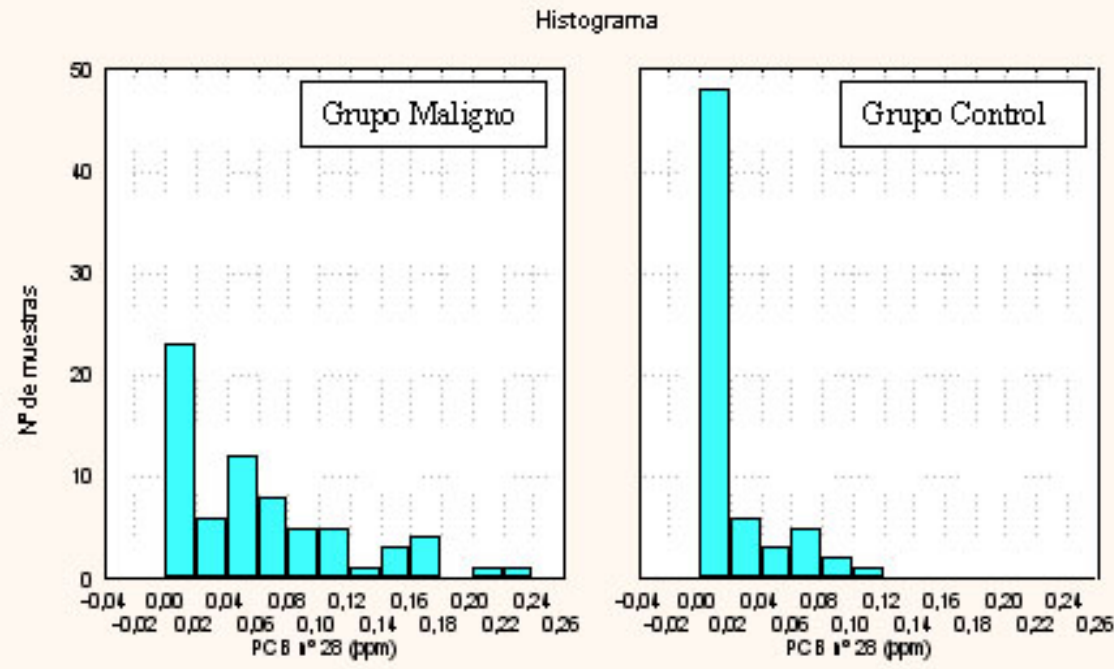

Grafica III - Distribución de frecuencia de las muestras según los grupos y concentraciones de PCB no 28. 
tre estos residuos y la malignidad de los tumores. En este sentido, Wassermann et al. $(1976)^{(19)}$ concluyeron que los niveles de compuestos organoclorados y PCBs en el tejido maligno se mostraron significativamente más elevados que los observados en el tejido de mama aparentemente normal. Wolff et al. (1993) ${ }^{(20)}$ encontraron una relación positiva entre el cáncer de mama y los niveles de DDE detectados en el suero de 58 casos con cáncer y 171 controles. El autor considera que las mujeres con altos niveles de DDE presentan 4 veces más riesgo de padecer cáncer de mama que las mujeres con bajos niveles de este metabolito. En la presente investigación, no se ha establecido una relación significativa en- tre la concentración de dicho compuesto en las muestras y el grado de patología de las donantes. Unger et al. (1984) $)^{(1)}$, Krieger et al. (1994) ${ }^{(4)}$ y Key y Reeves (1994) ${ }^{(21)}$ tampoco establecieron diferencias significativas en los nivels de DDE y PCBs entre los casos de cáncer y los controles. Refiriendose a los valores de p,p'-DDE determinados en este trabajo, las mayores distribuciones de muestras de ambos los grupos, se encuentran entre el límite de cuantificación (LD) y $2 \mu \mathrm{g} / \mathrm{g}$ de tejido mamario (Gráfica V). De entre las bibliografias consultadas, ninguna hace referencia a la Gráfica IV - Distribución de frecuencia de las muestras según los grupos y concentraciones de PCB n 52.

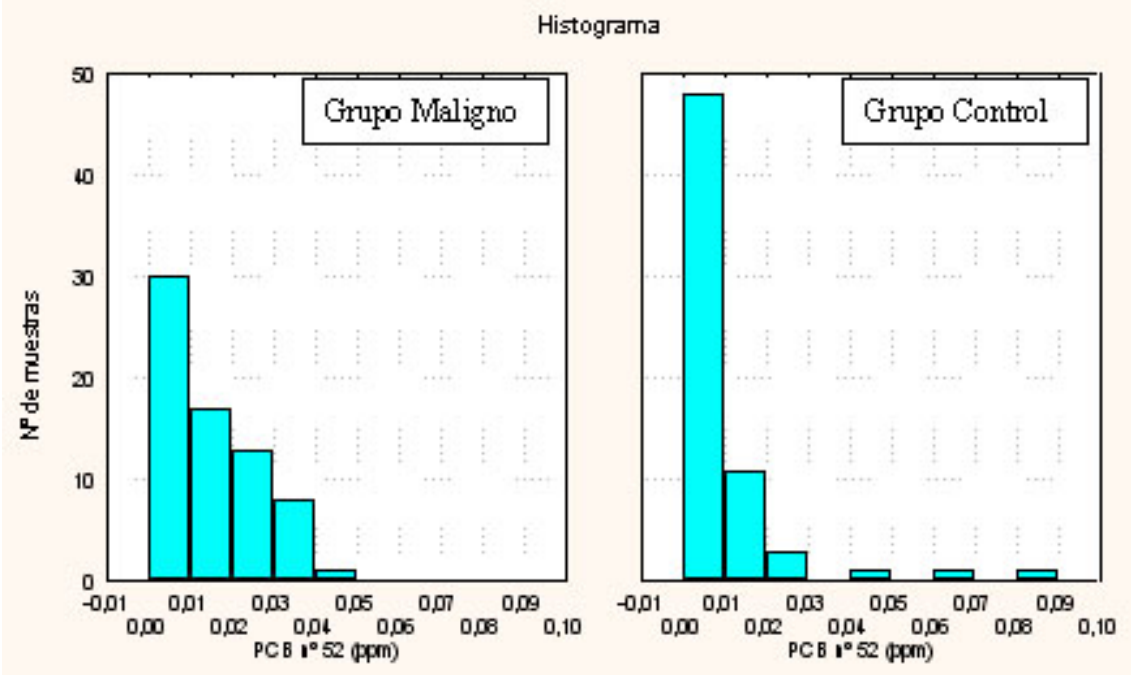

Gráfica IV - Distribución de frecuencia de las muestras según los grupos y concentraciones de PCB ํo 52.

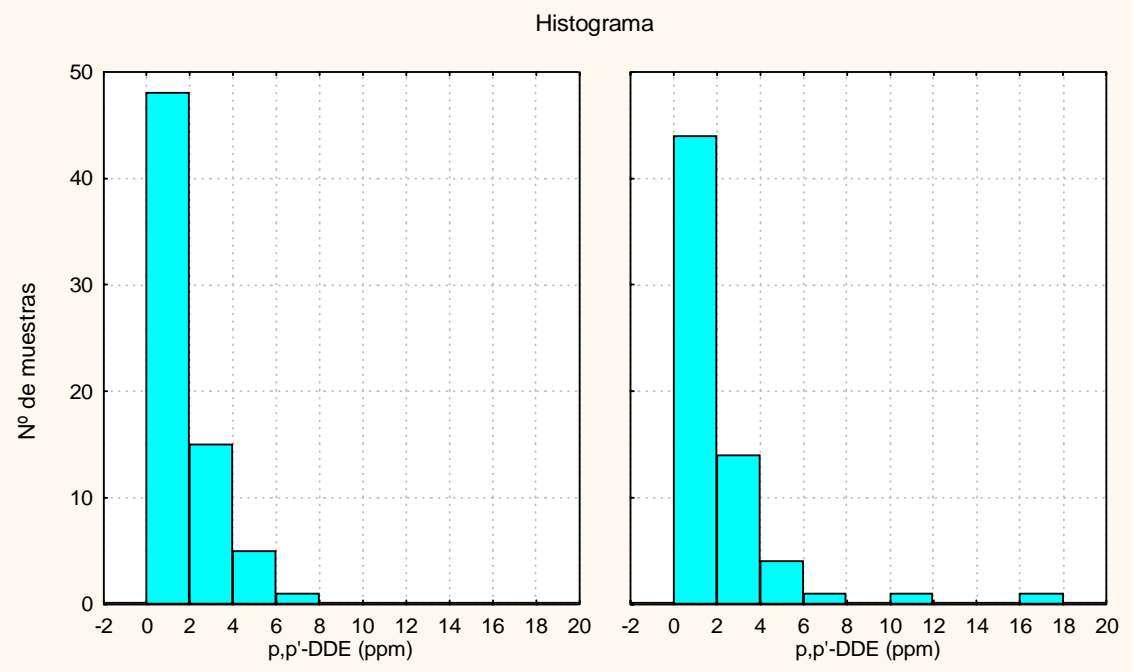

Gráfica V - Distribución de frecuencia de las muestras según los grupos y concentraciones de p,p'-DDE. 
Presencia de Aldrín en las muestras analizadas de tejido mamario de donantes que componían el grupo control o de donantes que componían el grupo con patología maligna, motivo por el cual no se ha podido comparar los resultados.

\section{b) Datos de las encuestas}

Los resultados obtenidos en los cuestio-narios aplicados a cada donante de muestra se reflejan en la Tabla II (datos cuantificables) y en la Tabla III (datos no cuantificables). Con los datos referentes al peso y a la talla, se ha calculado el índice de masa corporal (ICM), aplicando la seguiente fórmula:

$$
\mathrm{ICM}=\text { peso en } \mathrm{kg} /(\text { talla en } \mathrm{m})^{2}
$$

\begin{tabular}{|c|c|c|c|c|}
\hline \multirow{2}{*}{ Variables } & \multicolumn{2}{|c|}{ Valores Medios } & \multicolumn{2}{|c|}{ Desviación Típica } \\
\hline & Controles & Malignos & Controles & Malignos \\
\hline Edad & 45,8 & 56,6 & 14,320 & 16,053 \\
\hline Menarquia & 12,4 & 12,8 & 1,412 & 1,650 \\
\hline № de hijos & 2,1 & 2,5 & 2,142 & 1,820 \\
\hline Meses lactancia & 9,2 & 21,7 & 16,832 & 26,482 \\
\hline № lactancias & 1,8 & 2,0 & 2,105 & 1,818 \\
\hline Edad $1^{\mathrm{er}}$ parto & 24,8 & 24,4 & 4,662 & 3,312 \\
\hline № de abortos & 0,3 & 0,5 & 0,713 & 1,038 \\
\hline Menopausia & 47,2 & 48,2 & 6,890 & 4,600 \\
\hline Peso (kg) & 67,0 & 69,4 & 15,167 & 13,878 \\
\hline Talla (cm) & 158,6 & 155,5 & 6,040 & 7,000 \\
\hline IMC & 26,8 & 28,8 & 6,168 & 6,345 \\
\hline
\end{tabular}

Tabla III - Datos no cuantificables de las encuestas según el mayor percentual de las contestaciones de las donantes

\begin{tabular}{|c|c|c|}
\hline Datos & \multicolumn{2}{|c|}{ Mayor \% de contestaciones } \\
\hline Zona de nacimiento & Campiña y Córdoba capital & Campiña y Córdoba capital \\
\hline Zona de domicilio & Córdoba capital & Córdoba capital \\
\hline Estado civil & Casada & Casada \\
\hline Profisión actual & Sus labores & Sus labores \\
\hline Profisión anterior & Sus labores & Agricultura \\
\hline Antecedentes familiares & No había & No había \\
\hline Antecedentes personales & Sí tenían & No tenían \\
\hline Alimento más consumido & Frutas & Frutas \\
\hline Consumo semanal de pescado & 2 veces & $1 \mathrm{vez}$ \\
\hline Consumo semanal de carne & 3 veces & 3 veces \\
\hline Consumo diario de leche & $250 \mathrm{ml}$ & $250 \mathrm{ml}$ \\
\hline $\begin{array}{l}\text { Consumo de frutas, verduras y } \\
\text { legumbres }\end{array}$ & Toma a diario & Toma a diario \\
\hline Hábitos fumadores & Fuman menos de 10 cigarros por día & Fuman menos de 10 cigarros por día \\
\hline Hábitos alcohólicos & No bebedoras & No bebedoras \\
\hline $\begin{array}{l}\text { Contacto con productos químicos en } \\
\text { agricultura y ganadería }\end{array}$ & No fumigaron & No fumigaron \\
\hline Peso y sobrepeso & Peso normal & Obesidad leve y grave \\
\hline Procedencia de la muestra & Mama izquierda & Mama izquierda \\
\hline Voluman de la mama & Mediana & Mediana \\
\hline Localización de la lesión & Cuadrantes superiores & Cuadrantes superiores \\
\hline
\end{tabular}


Respecto a los datos cuantificables, la edad, los meses de lactancia, el índice de masa corporal y la talla son datos que presentaron significación estadística con relación a los grupos de donantes. Los tres primeros son más elevados en las donantes con patología maligna, mientras la talla es menor en las donantes de este grupo.

Los antecedentes personales y el peso y sobrepeso, clasificados como datos no cuantificables, son datos que presentaron también significación estadística con relación a los grupos de donantes. Respecto a los antecedentes personales, en el grupo control un mayor número de donantes había presentado anteriormente algún tipo de alteración en las mamas. En relación al peso y sobrepeso, las donantes con patología maligna presentaron mayores grados de obesidad.

\section{c) Relación datos encuestas/residuos organoclo- rados}

En lo que se refiere a la relación datos encuestas/residuos organoclorados, se observó que, con el aumento de la edad de las donantes, se produce un incremento de los niveles de residuos organoclorados en el tejido mamario, a excepción del Aldrín que presentó concentraciones más elevadas en las mujeres más jóvenes.

Teniéndose en cuenta la edad de las donantes del grupo control y la edad de las del grupo con patología maligna, los residuos organoclorados presentaron concentraciones más bajas en las mujeres más jóvenes de ambos los grupos. El Aldrín y los congéneres de bifenilos $\mathrm{n}^{\text {os. }} 28$ y 52 presentaron algunas particularidades. El Aldrín presentó concentraciones más altas en las mujeres más jóvenes del grupo control, mientras que en el grupo con patología maligna este contaminante presentó concentraciones más elevadas en las donantes mayores. El PCB $n^{\circ} 28$ presentó concentraciones más altas en las donantes del grupo maligno, independente de la edad. El PCB $\mathrm{n}^{\circ} 52$ presentó concentraciones más bajas en las mujeres más jóvenes del grupo control, y concentraciones más altas en las mujeres más jóvenes del grupo con patología maligna; este compuesto presentó concentraciones similares en las mujeres mayores de ambos los grupos de donantes.

Se establecieron correlaciones significativas entre los meses de lactancia y los niveles de residuos de HCB, Aldrín y PCBs nos. 28, 118, 138, 153 y 188. Estos compuestos presentaron concentraciones más elevadas en las donantes que lactaron durante más tiempo, y ello posiblemente se debe a la mayor edad que poseen estas donantes.

La talla se relacionó significativamente con los niveles de residuos de HCB, Aldrín y $\mathrm{PCB} \mathrm{n}^{\circ} 28$ detectados en las muestras de tejido mamario. Las donantes con menor talla presentaron concentraciones más elevadas de estos tres contaminantes.

El estudio antecedentes personales y niveles de residuos organoclorados presentó diferencias significativas para el p,p'-DDE y PCB no 118 . Los niveles de estos compuestos fueron superiores en las donantes que anteriormente habían presentado enfermedades.

También resultó significativo el estudio entre el peso y sobrepeso de las donantes y las concentraciones detectadas de HCB, p,p'-DDE y PCB n ${ }^{\circ} 118$. Estos tres contaminantes se encontraron en concentraciones más elevadas en las donantes con obesidades leve y grave.

\section{4- CONCLUSIÓN}

Los resultados obtenidos sobre el estudio de los cuestionarios indican que factores epidemiológicos, como la edad (50-60 años), tiempo de lactación (1030 meses), peso elevado y talla baja o alto índice de masa corporal, pueden ser considerados factores de riesgo con relación al cáncer de mama entre las mujeres cordobesas.

Los hábitos alimentarios de las donantes estudiadas no han sido significativos en relación a las donantes con patología mamaria maligna. Sin embargo, estos hábitos se relacionan parcialmente con los residuos organoclorados investigados, lo que posiblemente refleja la carga corporal de las donantes a los contaminantes detectados en sus muestras. En nuestra investigación se relaciona claramente el consumo de productos agrícolas, principalmente las verduras, con el compuesto mayoritariamente detectado en la grasa mamaria, el p,p'-DDE. La elevada concentración de p,p'-DDE en las muestras se atribuye a la larga persistencia de este compuesto en el medio ambiente y a la amplia utilización que ha tenido el DDT.

A la vista de las determinaciones realizadas respecto a cuatro pesticidas y once congéneres de bifenilos policlorados en este estudio, se observa una clara significación estatística de tres de estos compuestos (Aldrín, $\mathrm{PCB}{ }^{\circ} 28$ y $\mathrm{PCB} \mathrm{n}^{\circ}$ 52) que presentan mayores concentraciones en la grasa de mujeres con cáncer de mama; motivo por el que se concorda con 
las teorías actuales de que residuos organoclorados pueden actuar como promotores activos del cáncer de mama, si bien, y por ello, se hace imprescindible la realización de nuevas investi-gaciones con vistas a ampliar los conocimientos existentes sobre el tema abordado en esta investigación.

COSTABEBER I; ANGULO R \& JODRAL M. Organochlorate residues In mammary adipose tissue and their relation with to breast cancer. Medicina, Ribeirão Preto, 33: 506-514, oct./dec. 2000.

ABSTRACT: Taking into consideration the sanitary repercussion that organochlorate residues may have, levels of organochlorate pesticides and polychlorate biphenyls were determinated in samples of adipose tissue from donors with mammary cancer (group with malignant pathology) and beneficial alteration (group control). Furthermore, a study was made about these women epidemiologic data and, posteriorlly, the relation organochlorate residues/epidemiologic data was studed. The levels of Aldrin, PCB congener $n^{\circ} 28$ and PCB $n^{\circ} 52$ were statistically different in the control and malignant pathology groups. The mean concentrations of these compounds were lower in the control group than in the malignant pathology group, and this suggests the possibility of a positive relation between these residues and the malignancy of the tumours.

UNITERMS: Organochlorate Residues. Breast Neoplasms.

\section{REFERENCIAS BIBLIOGRÁFICA}

1 - UNGER M; KIAER H; BLICHERT-TOFT M; OLSEN J \& CLAUSEN $J$. Organochlorine compounds in human breast fat from deceased women with and without cancer and in biopsy material from newly diagnosed patients undergoing breast surgery. Environ Res 34: 24-28, 1984.

2 - MUSSALO-RAUHAMAA H; HASANEN E; PYYSALO H; ANTERVO K; KAUPILLA R \& PANTZAR P. Occurrence of betahexachlorocyclohexane in breast cancer patients. J Am Cancer Soc 66: 2124-2125, 1990.

3 - FALCK FJ; RICCI AJ; WOLFF MS; GODBOLD J \& DECKERS P. Pesticides and polychlorinated biphenyl residues in human breast lipids and their relation to breast cancer. Arch Environ Health 47: 143-146, 1992.

4 - KRIEGER N; WOLFF MS; HIATTRA; RIVERAM; VOLGELMAN $J$ \& ORENTREICH N. Breast Cancer and Serun Organochlorines: a Prospective Study Among White, Black, and Asian Women. J Natl Cancer Inst 86: 589-599, 1994.

5 - COSTABEBER I. Residuos organoclorados persistentes en grasa mamaria y su relación con los hábitos alimentarios: repercusiones sanitarias. Tesis Doctoral. Programa de Tecnología de los Alimentos. Facultad de Veterinaria. Universidad de Córdoba. España. 1999.

6 - AHLBORG UG; LIPWORTH L; TITUS-ERNSTOFF L; HSIEH CC; HANBERG A; BARON J; TRICHOPOULOS D \& ADAMI HO. Organochlorine compounds in relation to breast cancer, endometrial cancer, and endometriosis: An assessment of the biological and epidemiological evidence. Crit Rev Toxicol 25: 463-531, 1995.
7 - SAFE SH. Environmental and dietary estrogens and human health: Is there a problem?. Environ Health Perspect 103: 346-351, 1995

8 - PUJOL XG. Estrógenos: Amistades peligrosas. Cienc Vida 6: 104-109, Agosto 1998.

9 - GARRIDO FERNANDEZ MD; JODRAL VILLAREJO ML \& POZO LORA R. Bifenilos policlorados en leches esterilizadas españolas y estudio experimental del efecto de la temperatura sobre estos compuestos. $N+D$, № 0: 35-40, 1992.

10 - KANJA LW; SKAARE JU; OJWANG SBO \& MAITAI CK. A comparison of organochlorine pesticides residues in maternal adipose tissue, maternal bood, cord blood and human milk from mother/infant pairs. Arch Environ Contam Toxicol 22: $21-24,1992$

11 - LUDWICKI JK \& GORALCZYK K. Organochlorine pesticides and PCBs in human adipose tissue in Poland. Bull Environ Contam Toxicol 52: 400-403, 1994.

12 - GALLELLI G; MANGINI S \& GERBINO C. Organochlorine residues in human adipose and hepatic tissues from autopsy sources in northern Italy. J Toxicol Environ Health 46: 293-300, 1995.

13 - WALISZEWSKI SM; PARDIO SEDAS VT; CHANTIRI PEREZ JN; INFANZON RUIZ RM \& RIVERA J. Evaluación de los niveles de DDT y $\mathrm{HCH}$ en el tejido adiposo de algunas personas fallecidas en el Estado de Veracruz, México. Rev Int Contam Ambient 11: 87-93, 1995.

14 - KAMARINOS A; IOSIFIDOU EG; BATZIOS C; PSOMAS IE \& KILIKIDIS S. Residues of organochlorine pesticides and PCBs in human adipose tissues in Greece. Fresenius Environ Bull 6: 383-389, 1997. 
15 - SKAARE JU; TUVENG JM \& SANDE HA. Organochlorine pesticides and polychlorinated biphenyls in maternal adipose tissue, blood, milk and cord blood from mothers and their infants living in Norway. Arch Environ Contam Toxicol 17: 55-63, 1988.

16 - DUARTE-DAVIDSON R; HARRAD SJ; ALLEN S; SEWART AS \& JONES KC. The relative contribution of individual polychlorinated biphenyls (PCBs), polychlorinated dibenso-p-dioxins (PCDDs) and polychlorinated dibenzo-p-furans (PCDFs) to toxic equivalent values derived for bulked human adipose tissue samples form Wales, United Kigdom. Arch Environ Contam 24: 100-107, 1993.

17 - DUARTE-DAVIDSON R; WILSON SC \& JONES KC. PCB's and other organochlorines in human tissue samples from the welsh population: I-Adipose. Environ Pollut 84: 69-77, 1994.

18 - POLI A; VARISCHI G; ROLLO L; DE MARCO R \& CAMPELLO C. Contaminazione da policlorobifenil (PCB) del tessuto adiposo di soggetti della Provincia di Trento. Ann Ig 7: 8390, 1995.
19 - WASSERMANN MW; NOGUEIRA DP; TOMATIS L; MIRRAAP; SHIBATA H; ARIE G; CUCOS S \& WASSERMANN D. Organochlorine compounds in neoplastic and adjacent apparently normal breast tissue. Bull Environ Contam Toxicol 15: 478-484, 1976.

20 - WOLFF MS; TONIOLO PG; LEE EW; RIVERA M \& DUBIN N. Blood levels of organochlorine residues and risk of breast cancer. J Natl Cancer Inst 85: 648-652, 1993.

21 - KEY T \& REEVES G. Organochlorines in the environment and breast cancer. Br Med J 308: 1520-1521, 1994.

Recebido para publicação em 06/04/2000

Aprovado para publicação em 30/11/2000 